\title{
Data-Driven Inverse Modelling of Ionic Polymer Conductive Composite Plates
}

\author{
John G. Michopoulos ${ }^{1}$ and Moshen Shahinpoor ${ }^{2}$ \\ ${ }^{1}$ Special Projects Group, Code 6390.2, Center for Computational Material Science, \\ Naval Research Laboratory, USA \\ john.michopoulos@nrl.navy.mil \\ ${ }^{2}$ Artificial Muscle Research Institute School of Engineering and School of Medicine \\ University of New Mexico Albuquerque, NM 87131, USA \\ shah@unm.edu
}

\begin{abstract}
Analytical solutions of the partial differential equations (PDEs) governing the behavior of ionic polymer plates have not been yet obtained and therefore only time consuming discrete numerical methods can be used instead. To avoid the computational cost of numerical solutions this paper introduces a solution construction method that exploits analytical approximation basis functions borrowed from solutions of single physics formulations associated with rectangular ionic polymer plates for artificial muscle applications. This is achieved by utilizing an inverse approach that exploits global optimization. An objective function is constructed to express the error between the experimental and analytical values of the selected state variables. Minimization of this objective function yields an efficient determination of the unknown free coefficients. Comparisons between the determined approximations and the experimental data along with computational efficiency improvements conclude this paper.
\end{abstract}

\section{Introduction}

Recent progress on processing and development of various multi-field activated materials such as electro-active polymers (EAP) and ionic polymer conductive composites (IPCC) for artificial muscle applications [1,2, 3, has underlined two main needs. First, the general need for a rigorous, complete and preferably automated modelling methodology that will eventually accelerate the design, certification and prototyping of these materials and the devices or systems built around them. Second, the need for efficient exercise and application of the models derived from the previous methodology. We have already addressed the first need elsewhere [4, 5, 6, by following standard continuum multiphysics modeling approaches $7,8,9,10$. The present paper focuses on the second need. Thus, here an attempt to increase the computational efficiency of the process required to exercise models of rectangular plates constructed from various multi-field activated materials is presented.

Previous modeling efforts of EAP or IPCC plates [4,5] have resulted to a system of partial differential equations governing their multi-field behavior in space 
and time. This non-linear system is an extended Von-Karman set of equations. We have demonstrated [4, 5, 6, that this system is solvable via the the use of the Finite Element Method (FEM). However, the time required to obtain FEM solutions has been hindered by two main difficulties. First, a general couple PDE system solver code is required by the investigator at hand. There are commercial codes (i.e. "flexPDE" 11, "COMSOL Multiphysics" 12]) and open source free codes (i.e. "freeFEM++" and "freeFEM3D" 13]) that may exceed the user's monetary and/or time (for learning how to use it) investment capability. Second, -assuming that a user owns a code of this type-, the computational cost can me prohibiting for specific applications especially because the non-linear and dynamic character of the associated PDEs.

The goal of this paper is to develop a method that alleviates the second of these problems by introducing a methodology that avoids the discretization and the numerical integration of the associated PDEs. The proposed method has been inspired by the overall "data-driven " framework associated with the development of a general computational environment for multiphysics applications (DDEMA) that has been preliminarily described elsewhere [14, 15].

The novelty of this paper is three fold. First, it avoids the need to integrate the multiphysics PDEs describing the dynamics of the system all together. Second, it proposes a technique based on approximating the solution fields by sums of basis functions borrowed from well known single-physics approximations. Third, it casts the problem as an optimization problem where an objective function is constructed that expresses the norm of the error vector between the experimental values of the fields involved in the problem and their respective analytical approximations constructed as previously described.

The paper presents the coupled and the partially uncoupled multiphysics formulations for a rectangular IPCC plate in Section 2. It subsequently describes the experimental procedure for acquiring the data to be utilized in Section 3 . The corresponding global optimization problem is being setup and applied and validated in section 4 . Conclusions provide the closure of the paper.

\section{Multi-field Coupled and Uncoupled System of PDEs}

We have demonstrated in the past [4, 5, 6] that introduction of Lagrange strains (to account for large deformations) into the general theory of multi-component species diffusion of electro-thermo-elastic continua, with insignificant ionic currents and chemical reactivity, excited by generalized loading conditions of multi-component mass concentrations in the boundaries, electric potential, temperature and mechanical deformation yields the following set of modified VonKarman nonlinear PDEs :

$$
\begin{gathered}
\nabla^{2} \nabla^{2} w+(1+\nu) \sum_{k} \nabla^{2} X^{k}=\frac{h}{N}\left(\frac{q}{h}+F_{, 22} w_{, 11}-2 F_{, 12} w_{, 12}+F_{, 11} w_{, 22}\right), \\
\nabla^{2} \nabla^{2} F+E \sum_{k} \nabla^{2} X^{k}=E\left[\left(w_{, 12}\right)^{2}-w_{, 11} w_{, 22}\right],
\end{gathered}
$$




$$
\frac{1-\nu}{2 E}\left(\nabla^{2} \delta_{i j}-\partial_{i} \partial_{j}\right) F \delta_{i j}+\nabla^{2} X^{k}=0
$$

Here $w, F, X^{k}$ are the deflection, the Airy stress function and the additional generalized field state variables and $\delta_{i j}, \nu, E, h, N, q$ are Kronecker's delta, Poisson's ratio, the modulus of elasticity, the plate thickness, the flexural rigidity and the distributed load respectively.

When all non-mechanical fields $X^{k}$ are not present, this system reduces to the traditional system of the large deflection Von-Karman equations. Analytical approximation solutions of this system are to be used for solving Eqs. (11).

\section{Experimental Procedure for Data Collection}

To acquire preliminary experimental data reflecting the electrically activated bending of IPCC plates, an IPCC square specimen was cut with dimensions $50 \times$ $50 \times 0.3 \mathrm{~mm}$, where the small number corresponds to the the thickness of the plate while the other two numbers correspond to the length and width of the plate. Figure 1(a) presents a view of the IPCC plate mounted on conductive frame that was used to apply voltage boundary conditions along the entire boundary. In Fig. 1(b) the experimental setup shown including a load cell used to determine the deflection at the mid-point of the plate surface. The load cell is mounted on a precision screw type platform capable of applying measurable displacement towards and away from the center of the plate. Consecutive turns of the screw were applied all corresponding to a displacement of a about a micron. For each one of these turns, when zero contact force was first observed (the load cell was not touching the plate) the distance traveled by the load cell becomes equal to the deflection of the plate.

Figure 2(a) shows the experimental results of applying a sinusoidally varying voltage and the corresponding deflection at the middle of the plate as a function of time. Figure 2(b) shows the voltage vs. the mid-point deflection for the first two seconds. These are the data to be used in the process that follows.

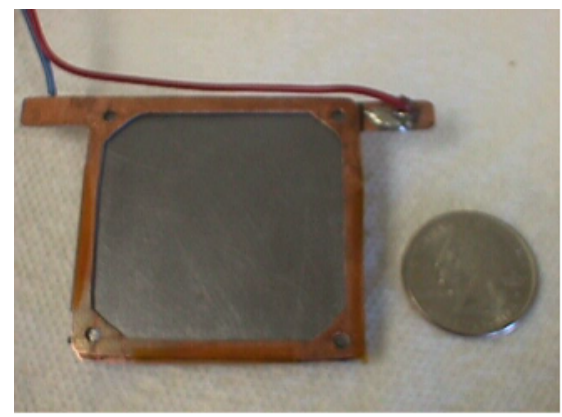

(a)

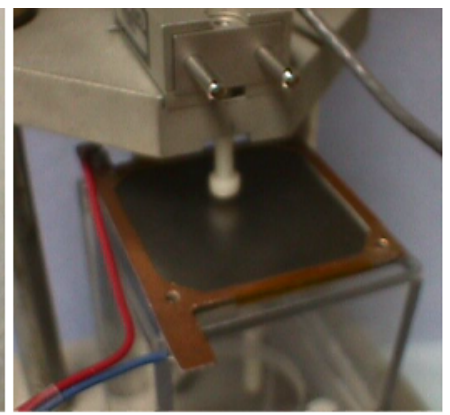

(b)

Fig. 1. Closeup view of Square IPCC plate mounted on conductive frame (a), and experimental setup of loading frame with loading cell in place (b) 


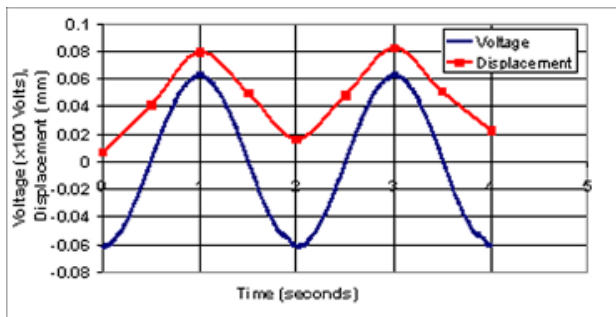

(a)

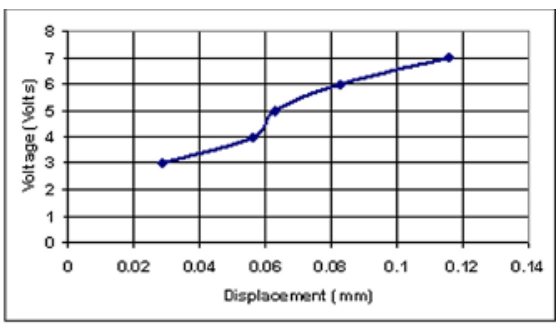

(b)

Fig. 2. Voltage and mid-point deflection vs. time (a), and voltage vs. mid-point deflection (b)

\section{Global Optimization for Analytical Approximation of Simulated Behavior}

The inverse approach character of the proposed methodology is based on minimizing in the least-squares sense the objective function

$$
f^{o}\left(c_{i j}\right)=\left\{\sum_{i=1}^{n}\left[w_{i}^{s}\left(c_{1 j}\right)-w_{i}^{e}\right]^{2}+\sum_{i=1}^{n}\left[F_{i}^{s}\left(c_{2 j}\right)-F_{i}^{e}\right]^{2}+\sum_{i=1}^{n}\left[V_{i}^{s}\left(c_{3 j}\right)-V_{i}^{e}\right]^{2}\right\},
$$

where $w_{i}^{s}\left(c_{1 j}\right), F_{i}^{s}\left(c_{2 j}\right), V_{i}^{s}\left(c_{3 j}\right)$ are the unknown variables of the simulated fields corresponding to the deflection, Airy stress function and voltage distributions; $w_{i}^{e}, F_{i}^{e}, V_{i}^{e}$ are the corresponding experimental values of the same variables respectively. Here, the unknowns to be determined by the minimization of Eq. 2 are the free coefficients $c_{i j}$.

In our preliminary experimental implementation the Airy stress function and the voltage distribution over the domain of the space variables $(x, y)$ were not measured. Therefore, only the first term of Eq. 2 (corresponding to the deflections) was used in the actual numerical analysis. This implies that $c_{i j}=c_{1 j}=c_{j}$.

We constructed the simulated deflection solution $w_{i}^{s}\left(c_{1 j} ; x_{i}, y_{i}\right)$ indexed for specific locations $\left(x_{i}, y_{i}\right)$ as an additive composition of just three basis functions $\tau_{j}$ with, $(j=1,2,3)$, that satisfy the boundary conditions of zero deflection along the edges according to

$$
w_{i}^{s}\left(x_{i}, y_{i}\right)=\frac{4 q^{\text {equiv }} a^{4}}{\pi^{5} D}\left[c_{1} \tau_{1}\left(x_{i}, y_{i}\right)+c_{2} \tau_{2}\left(x_{i}, y_{i}\right)+c_{3} \tau_{3}\left(x_{i}, y_{i}\right)\right]+w_{i}^{\text {off set }},
$$

where $D$ is the flexural rigility of the plate, $q^{\text {equiv }}$ is an equivalent lateral load distribution per unit of area of a small deflections plate that generates deflections identical to our multi-filed plate, and $w_{i}^{\text {offset }}$ was added to capture the possible existence of any initial deflection. The three free coefficients $c_{j}$, weighting the basis functions are the unknowns to be determined.

Based on the infinite series analytical solutions constructed for approximating a solution satisfying the biharmonic equation governing the single physics small 
deflection bending of rectangular plates given in the past, we chose our basis functions to consist of the first three terms from one of them [16] according to

$\tau_{m}\left(x_{i}, y_{i}\right)=\cos \frac{m \pi x_{i}}{a}\left(1-\frac{\alpha_{m} \tanh \alpha_{m}+2}{2 \cosh \alpha_{m}} \cosh \frac{m \pi y_{i}}{a}+\frac{1}{2 \cosh \alpha_{m}} \frac{m \pi y_{i}}{a} \sinh \frac{m \pi y_{i}}{a}\right)$

with $\alpha_{m}=m \pi b / 2 a$ and where $a, b$ are the dimensions of the plate along the $x, y$ axes respectively.

Since the experimental data for the deflection are collected only at the midpoint of the plate we used another solution of an equivalent single physics approximating solution of the biharmonic equation to generate pseudo experimental data for the rest of the points on the plate according to,

$$
\begin{array}{r}
w_{i}^{e}\left(x_{i}, y_{i}\right)=\frac{16 q^{\text {equiv }}}{\pi^{6} D} \sum_{m=1}^{100} \sum_{n=1}^{100} \frac{a^{4} b^{4}}{m n\left(b^{2} m^{2}+a^{2} n^{2}\right)^{2}} \\
\sin \frac{m \pi\left(2 x_{i}+a\right)}{2 a} \sin \frac{m \pi\left(2 y_{i}+b\right)}{2 b}+ \\
+w_{i}^{\text {offset }}+w_{i}^{\text {gaussian_noise }},
\end{array}
$$

where $w_{i}^{\text {gaussian_noise }}$ represents a term injecting noise for the purpose of emulating the error due to the experimental data-acquisition methodology. It is worthwhile mentioning here that the fact that we use only the first 100 terms of the double series in the relation above is due to the fact that we are not concerned to capture accurately the deflection of the plate but rather in gross manner. The injected noise is expected and has been chosen to be of equal or higher magnitude than the approximation error introduced from not considering more terms.

To eliminate the presence of the equivalent mechanical load distribution from Eqs. 3 and 5 the Voltage vs. mid-point (maximum) deflection observed in Fig. 2(b) has been approximated by the following second order polynomial

$$
w^{\max }=0.00000137903 V_{o}^{2}+0.00000635696 V_{o}+0.000000234504 .
$$

Equating this deflection with that provided by Eq. 3 at the midpoint, and solving the resulting equations with respect of $q^{\text {equiv }}$ we obtain the following voltage depended solution for

$$
q^{\text {equiv }}=\frac{\pi^{5} D}{4 a^{4}} \frac{\left(0.00000137903 V_{o}^{2}+0.00000635696 V_{o}+0.000000234504-w_{i}^{\text {off set }}\right)}{\left[c_{1} \tau_{1}(0,0)+c_{2} \tau_{2}(0,0)+c_{3} \tau_{3}(0,0)\right]} .
$$

Equations 3 ,7 fully define the quantities participating in Eq2 and therefore determination of the unknown parameters $c_{j}$ can be achieved by using any quadratic programming methodology for minimizing Eq. 2, In the context of this work the implementation of the optimization procedure was formulated in Mathematica [17] via the algorithms that were available in the package Global Optimization "GO-5.0" [18]. The resulting solution is presented in Table 1] and the three coefficients are compared with the known values of the first 3 terms of the infinite series that approximates the biharmonic solution. The computational time for determining these coefficients such that the objective function 
Table 1. Computed solution coefficients $c_{i}$ and its comparison with know terms of the single physics solution

\begin{tabular}{|c||c|c|c|}
\hline & $c_{1}$ & $c_{2}$ & $c_{3}$ \\
\hline \hline Solution & 1.00000 & 0.00432335 & 0.00000310941 \\
\hline Known Terms & 1.00000 & 0.00411523 & 0.00032 \\
\hline
\end{tabular}

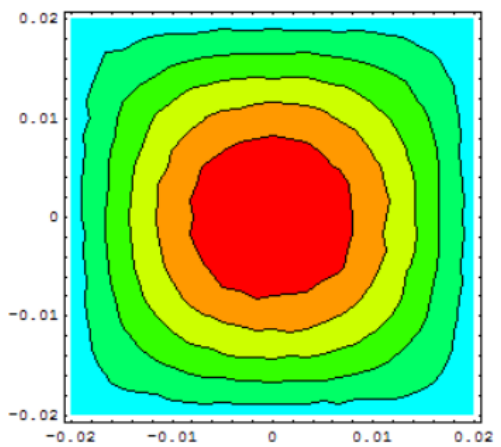

(a)

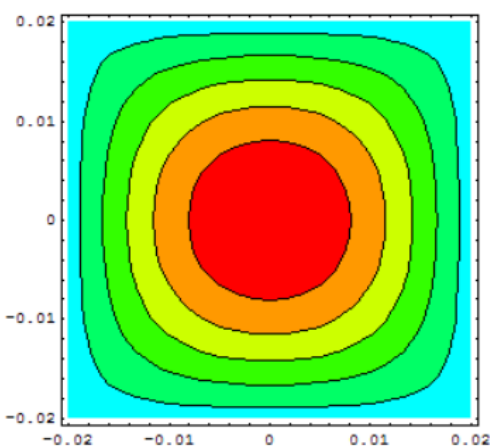

(b)

Fig. 3. Deflection field distributions for the pseudo-experimental case (a), and the analytically computed one (b)

given by Eq. 2 is minimized, for a problem that evaluates the simulated and experimental values of deflection at 25 points of the plate, on a Pentium IV 3.2 $\mathrm{GHz}$ machine was just under 53 seconds. Now that $c_{j} \mathrm{~s}$ are known the deflection given by Eq. 3 is fully determined. For comparison purposes Fig. 3 shows the experimental (left) and simulated (right) distributions of the displacement field over the square plate at the same arbitrary time moment.

Clearly, the computationally determined simulated solution tracks the pseudo-experimental one very well. The jaggedness of the contours in the pseudoexperimental deflection distributions shown in Fig. 3(a) is due to the noise introduced in Eq. 5 .

The computational cost of such a distribution via Eq. 3 is about 0.005 seconds while the respective solution due to the appropriate finite element analysis takes an average of 195 seconds. The speedup factor is therefore $195 / 0.005=39,000$. Even if one includes the time for applying the optimization process to determine the coefficients $c_{j}$ the speedup factor is $195 /(53+0.005)=3.67$. The advantage of using a data-driven approximation of the unknown deflection field in place of a numerical discretization solution of the associated PDEs via FEM is obvious.

\section{Conclusions}

In this effort, we have described a methodology for determining partial analytical representations approximating the solution of the multi-field PDEs governing the 
behavior of the large deflection generalized Von-Karman plates constructed from IPCC. This is achieved by exploiting single physics (just mechanical) solutions of the biharmonic PDE governing small deflection plates to construct analytical approximations for the simulated deflection field. An optimization scheme for minimizing an objective function constructed in the least square sense as a measure of the square difference between the simulated and experimentally acquired (and pseudo-experimentally constructed) deflection field. The resulting solution tracks very well the experimental fields. The realized speedup factors are significant and justify the usage of this approach.

An essential limitation of this approach is that due to lack of experimental measurements for the spatial distribution of the Airy stress function and the voltage fields, no approximations for these fields has been derived. Future efforts will address this limitation. Once this is done then the PDEs themselves can further restrict the coefficients of the approximating solutions and thus further simplify their form. In addition, instead of only considering approximation solution of the small deflection plate theory this method can be extended to include approximation solutions of the large deflection theory of Von-Karman equations [19, 20]. Another, needed activity is a sensitivity analysis to classify how the increase of the number of the basis functions participating in the solution effects both the accuracy and the computational time of the entire process.

Acknowledgement. The authors acknowledge the support by the National Science Foundation under grants EIA-0205663 and CNS-0540419. Partial support from NRL's 6.1 core-funding is also greatly acknowledged.

\section{References}

1. Kim K. J. and Shahinpoor M., Ionic Polymer-Metal Composites II. Manufacturing Techniques, Smart Materials and Structures (SMS), Institute of Physics Publication, 12(1) (2003) 65-79.

2. Kim K. J. and Shahinpoor M., Ionic Polymer-Metal Composites III. Modeling and Simulation As Biomimetic Sensors, Actuators, Transducers and Artificial Muscles, Smart Materials and Structures Int. J., 13(4), (2004) 1362-1388.

3. Shahinpoor M. and Kim K.J., Ionic Polymer-Metal Composites IV. Industrial and Medical Applications, Smart Materials and Structures Int. J., Smart Mater. Struct. 14(1), (2005)197-214.

4. Michopoulos, J.G., Shahinpoor, M., Towards a Multiphysics Formulation of Electroactive Large Deflection Plates Made from Ionic Polymeric Artificial Muscles, Proceedings of First World Congress on Biomimetics and Artificial Muscles, 9-11 December 2002, Albuquerque NM.

5. John G. Michopoulos, Generalized multifield Von-Karman equations for large deflection of artificial muscle plates, Proc. SPIE Vol. 5387, Smart Structures and Materials 2004: Active Materials: Behavior and Mechanics; Dimitris C. Lagoudas; Ed., (2004), 12-23. 
6. John Michopoulos, "Computational Modeling of Multi-Field Continuum Systems," Proc., International Conference Computational Science - ICCS 2004, Krakw, Poland, June 6-9, 2004, Part IV Series: Lecture Notes in Computer Science, Vol. 3039 - ICCS 2004 Bubak, M.; Albada, G.D.v.; Sloot, P.M.A.; Dongarra, J. (Eds.), LNCS 3039, (2004), 621-628.

7. Green, A. E., Naghdi, P. M., A unified procedure for construction of theories of deformable media. I. Classical continuum physics, Proc. Roy. Soc. London Ser. A 448 /1934, (1995), 335-356.

8. Eringen, A.C., Maugin., G.A., Electrodynamics of Continua I. Foundations and Solid Media, Springer-Verlag, New York, 1990.

9. Sih, G.C., Michopoulos, J.G., Chou S.C., Hygrothermoelasticity, Martinus Nijhoff Publishers (now Kluwer Academic), 1986.

10. Michopoulos, J.G., Charbel Farhat, Jacob Fish, Modeling and Simulation of Multiphysics Systems, Journal of Computing and Information Science in Engineering - September , 5(3), (2005), 198-213.

11. PDE Solutions Inc., FlexPDE 5 Reference manual, PDE Solutions Inc., URL: http://www.pdesolutions.com/, (2005).

12. COMSOL Inc., COMSOL Multiphysics 3.2 Reference manual, COMSOL Inc. , URL: http://www.comsol.com/products/multiphysics/, (2006).

13. Ionut Danaila, Frdric Hecht, Olivier Pironneau, freeFEM++ and freeFEM3D distributions at URL: http://www.freefem.org/, (2005).

14. Michopoulos, J., Tsompanopoulou, P., Houstis, E., Rice, J., Farhat, C., Lesoinne, M., Lechenault, F., DDEMA: A Data Driven Environment for Multiphysics Applications,in: Proceedings of International Conference of Computational Science ICCS'03, Sloot, P.M.A., et al. (Eds.) Melbourne Australia, June 2-4, LNCS 2660, Part IV, Springer-Verlag, Haidelberg, (2003) 309-318.

15. Michopoulos, J., Tsompanopoulou, P., Houstis, E., Farhat, C., Lesoinne, M., Rice, J., Joshi, A., On a Data Driven Environment for Multiphysics Applications, Future Generation Computer Systems, 21(6), (2005), 953-968.

16. Timoshenko, Stephen P.; Woinowsky-Krieger, S., "Theory Of Plates And Shells," Engineering Societies Monographs, New York: McGraw-Hill, (1959).

17. Wolfram, S.,1999, The Mathematica Book, 4th Edition, Wolfram Media, Cahmpaign and Cambridge University Press, Cambridge.

18. Loehle Enterprises, Global Optimization 5.0 for Mathematica, Loehle Enterprises, (2004).

19. vonKarman, T., 1910, Festifkeitsprobleme im Maschinebau, Encyk. der Math. Wiss., pp. 349.

20. Levy, S., 1942, Bending of Rectangular Plates with Large Deflections, NACA Technical Report No. 737. 